\title{
Hysteresis loss in Brushless Doubly Fed Induction Machines
}

\author{
M. Ahmadian, B. Jandaghi and H. Oraee \\ Center of Excellence in Power System Management and Control, \\ Department of Electrical Engineering, Sharif University of Technology \\ Azadi Ave., Tehran, Iran \\ Phone/Fax number: +98935460 6644, e-mail: mhd.Ahmadian@gmail.com, Behzad_Jandaghi@ee.Sharif.edu, \\ Oraee@sharif.edu
}

\begin{abstract}
Brushless doubly fed machine (BDFM) is a potential for future wind energy generation, due to its lower maintenance costs and higher reliability than conventional doubly fed systems. While efficiency and design optimization of the machine has become critical issues recently, no analytical core loss model is investigated in the literature. Furthermore, core loss modeling is different from conventional induction machines, because of double frequency excitation of the machine. In this paper, a dynamical system is used for modeling of hysteretic behavior of iron core, and hysteresis losses of BDFM are analyzed and modeled mathematically. The flux waveforms of BDFM are assumed pure double sine, as in wound rotor BDFM lower harmonic values than nested loop design exist.
\end{abstract}

\section{Key words}

Brushless doubly fed machine (BDFM), core losses, hysteresis loop, dynamical systems, curve fitting.

\section{Introduction}

In standard frequencies $(50$ or $60 \mathrm{~Hz})$, core losses are around 20-25 percent of losses in electrical machines [1]. In BDFM, the amount of core loss is increased due to poor magnetic design [2]. Since this machine has two supply frequencies (power and control windings), definition of operating point is fairly different with single fed machines, so there are more different parameters for designing the machine. Until now, there is no contribution on mathematical calculation of losses in BDFM to be used in optimization of machine design or operating point, except the simulation of the machine by Ferreira et al [3], using 2D finite element method. In the simulation, the nonlinearity and saturation effects of iron core are taken into account. To calculate core losses, hysteresis and eddy current losses are considered, but hysteresis loss is calculated using classic models of conventional singly fed machines, where single sinusoidal supply exists. However, this is not compatible with flux distribution of a BDFM.

Core losses are generally divided into hysteresis, classic eddy, and excess eddy current losses. For each of them, there are certain dynamic and mathematic relations. In this paper, hysteresis loss of a BDFM is investigated. To do this, core flux is assumed 2D, to simplify flux calculation.

\section{Hysteresis Losses}

There are three main ideas for modeling the hysteresis systems. First method is fundamental analysis based on physical principles of the system, resulting in a large dynamic system [4], [5]. Although the method is the most accurate model, modeling comprehensive internal mechanisms of the system is mandatory, so it is complicated. Even, if the accurate model of the system is obtained, it should be simplified for computer simulations.

The second approach utilizes static models extracted from interpolation of experimental data. This method is generally used for sinusoidal supply systems and is widely used in electrical engineering systems. The main goal of these methods is to calculate energy losses in hysteretic systems. In fact, these models cannot simulate the hysteresis loops, and only contribute an estimate for the surface of the hysteresis loop. The waveforms studied in these models, are quasi-sine waves with little distortion from fundamental harmonic, separating the hysteresis loop into major and minor loops. These methods are based on estimation of surface of major and minor loops, as a function of waveform peak values. The most familiar nonlinear model is found in [6], introducing area of the hysteresis loop as an algebraic function of the peak flux density. The exponent is a constant number, which varies by the material. There are more accurate exponential models, which have the term of peak of flux density in their exponents [7]. In some of these models, exponent is a linear function of magnetic flux density [8], and in some others, quadratic function is preferred [9]. These models are mainly applied to single sinusoidally excited cores and the constants are calculated by curve fitting approach.

The third approach combines the two ideas to obtain a macroscopic view of hysteresis dynamics. This mathematical modeling approach can be verified by analogy of experimental and theoretical properties of the system. Since the hysteresis phenomenon is nonlinear, the model should be nonlinear either. If the model was linear, the accuracy of the model could be evaluated by a single experiment, according to Representation Theorem [10]. For experimental evaluation of the nonlinear model, infinite experiments are required, and the system cannot be evaluated by a single experiment anymore [11]. So, the model is evaluated qualitatively, by means of assessment of the main characteristics of the real system and the model. Then the model parameters and functions can be determined by experimental data. According to 
the analogy of the qualitative properties of both systems, it is expected that behaviour of the system in various excitations be accurate. The assumption is investigated by many different experiments in practice. Volterra proposed an integro-differential equation as the first step in developing the model [12]. Unfortunately, his model did not introduce a proper method for derivation of the kernel of the equation. To solve this problem, Block [13] and De Figueiredo [14] presented a Function Space method and employed piecewise-linear techniques. After them, Resh presented a model based on the similarities of the hysteresis phenomenon and a specific mechanical system, however, the model was very complicated [15].

The model of this paper is based on the Volterra's equation, but in contrast to it, an applicable approach for extraction of functions and model parameters is appended. A key advantage of this model is its overall simplicity, which is a valuable benefit in modelling of large systems.

Although the second modelling method is very typical in electrical engineering systems, it is not appropriate for modelling hysteretic dynamics of fields in a BDFM. First reason is the labyrinth of minor loops which their area cannot be calculated independently. Furthermore, in some situations, hysteresis loops are not symmetric, so the positive and negative peaks are not equal. In accordance with these reasons, using the third method is preferred.

\section{Hysteresis Loss Mathematical Model}

In [11], a dynamical system is proposed to model hysteretic relationship of the variables $x$ and $y$ :

$$
\frac{d y(t)}{d t}=g[x(t)-f(y(t))]
$$

where, $f$ and $g$ are strictly monotonically increasing, and differentiable functions, with nonzero slopes throughout the entire real line, and $g(0)=f(0)=0$.

It can be shown that the function $g$ corresponds with magnetic losses, while the function $f$ represents for the energy storage mechanism. Hence the $g$ and $f$ functions are called as "dissipation function" and "restoring function" respectively. Functions $f$ and $g$ can be determined to simulate a real system, using simple geometric calculations on a couple of system waveforms $x(t)$ and $y(t)$. It can be shown that, increasing the supply frequency will increase the area of the hysteresis loop. This phenomenon is called "widening effect". The model can simulate minor loops properly.

\section{Determination of dissipation restoring functions}

and

As discussed above, the presented dynamic model has the majority of important characteristics of the real hysteresis systems, so the system parameters can be defined based on a certain hysteresis loop, that the model can simulate the real system in other situations. Now we should present a method to define "dissipation function" and "restoring function". To do so, we should first measure the $\{x(t), y(t)\}$. The supply waveforms can be chosen arbitrarily. The simplest waveform to excite the system is a cosine excitation for $y(t)$. Therefore, $y(t)$ is an even function and $y^{\prime}(t)$ is odd. Each $x(t)$ waveform can be split into an even and an odd component as below:

$$
x(t)=x_{e}(t)+x_{o}(t)
$$

If the system can be assumed symmetrical, in other words, $f$ and $g$ are odd; combining (1) and (2) we have:

$$
x_{e}(t)=f(y(t)), \quad x_{o}(t)=g^{-1}\left(y^{\prime}(t)\right)
$$

Since $y(t)$ is sinusoidal, there exist two instants of time that:

$$
y\left(t_{1}\right)=y\left(t_{2}\right) \quad t_{1}, t_{2} \in[0, T]
$$

and

$$
y^{\prime}\left(t_{1}\right)=-y^{\prime}\left(t_{2}\right)
$$

Considering the $g$ is odd, we have:

$$
g^{-1}\left(y^{\prime}\left(t_{1}\right)\right)=-g^{-1}\left(y^{\prime}\left(t_{2}\right)\right)=d
$$

With substituting (6) in (3), we will have:

$$
x_{e}\left(t_{1}\right)=-x_{o}\left(t_{2}\right)=d
$$

Since $\left\{x\left(t_{1}\right), \mathrm{y}\left(t_{1}\right)\right\}$ and $\left\{x\left(t_{2}\right), y\left(t_{2}\right)\right\}$ are two points with the same value on the $y$ axis we can write:

$$
x_{e}\left(t_{1}\right)=-x_{o}\left(t_{2}\right)=\frac{x\left(t_{1}\right)-x\left(t_{2}\right)}{2}=d
$$

It can be seen in Fig. 1 that $d$ is the distance between the edges and the median of the hysteresis loop. Furthermore, it is concluded from the (3) that the median of the hysteresis loop satisfies the below equation:

$$
y\left(t_{1}\right)=y\left(t_{2}\right)=f^{-1}\left(x_{e}\left(t_{1}\right)\right)=f^{-1}\left(x_{e}\left(t_{2}\right)\right)
$$

So the median demonstrates the $f$ function. Considering that $y(t)$ is cosine, from the point $y(t)=0$ to the point where the $y(t)$ becomes maximum, $y(t)$ will be strictly decreasing. For the $g^{-1}$ function, this means that with an increase of $y(t)$, the hysteresis loop is narrowing. The details of the determination process of the $f$ and $g$ functions are shown in Fig. 1. It is worth noting that $f$ and $g$ are unique functions.

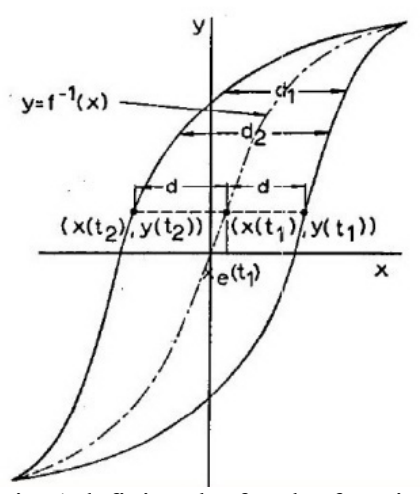

Fig. 1 defining the $f$ and $g$ functions 
If the hysteresis loops and the waveforms were not symmetric, we can use other methods such as numerical optimization to extract the functions.

\section{Accuracy of the dynamic model}

A sample of simulation of the model is portrayed in Fig. 2 and accuracy of the model in estimating the complicated loops is verified [11].

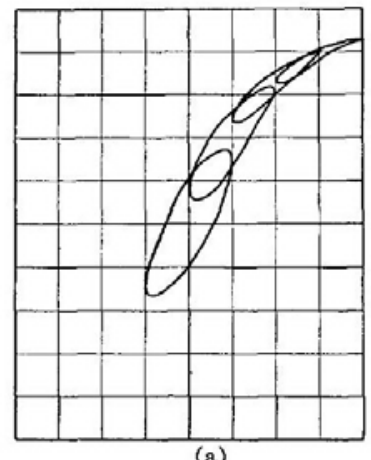

Fig. 2 hysteresis loops (a) experimental (b) simulation
Fig. 2 (a) shows the experimental hysteresis loop and the Fig. 2 (b) shows the simulation result of the hysteresis loop by the model [11]. We can see that the model has simulated the real system accurately.

\section{Hysteresis Loops in a BDFM and Corresponding Losses}

In a BDFM, rotor and stator field variations should be taken into account. In synchronous operation of the BDFM, the rotor field has a single frequency (synchronous frequency $\omega_{s}$ ). The stator field, consists of two fields, power winding and the control winding, with the frequencies of $\omega_{1}$ and $\omega_{2}$, respectively. In [16] the magnetic field of a BDFM in the air gap is stated as:

$$
B(\theta)=B_{1} \operatorname{Cos}\left(\omega_{1} t-p_{1} \theta\right)+B_{2} \operatorname{Cos}\left(\omega_{2} t-p_{2} \theta+\gamma\right)
$$

transformation of the above equation to rotor's reference results in:

$$
\begin{gathered}
\theta=\omega_{r} t+\varphi \\
B_{r}(\varphi)=B_{1} \operatorname{Cos}\left(\omega_{s} t+p_{1} \varphi\right)+B_{2} \operatorname{Cos}\left(\omega_{s} t+p_{2} \varphi+\gamma\right)
\end{gathered}
$$

Due to different field waveforms, stator and rotor have different amount of losses. In each $\delta \theta$ section of the machine, magnetic field of machine can be calculated. So to calculate the whole losses, amount of loss is calculated with respect to $\theta$, then integrated on interval $[0,2 \pi]$. This is equivalent to calculating the average losses with respect to $\theta$, then multiplying the result with $2 \pi$. This procedure is used in the numerical calculation algorithm. Another important point is that the machine losses are independent of phase difference of the fields, if the slip speed is not zero. The evidence of the assert is that with proper time and angle shifts of the magnetic field, the field distribution, corresponding to $\gamma=\gamma_{1}$ and $\gamma=\gamma_{2}$, are the same. Since calculation the losses is performed by averaging on time and angle, time and angle shifts do not change the final result.

Lemma 1: the machine losses are independent of phase difference of the fields, if the slip speed is unequal to zero.

Proof. If two fields with different phase values are assumed, the time and angle shifts are performed to fit them over each other:

$$
\begin{gathered}
B_{I}(\theta, t)=B_{1} \operatorname{Cos}\left(\omega_{1} t-p_{1} \theta\right)+B_{2} \operatorname{Cos}\left(\omega_{2} t-p_{2} \theta+\gamma_{1}\right) \\
B_{I I}(\theta, t)=B_{1} \operatorname{Cos}\left(\omega_{1} t-p_{1} \theta\right)+B_{2} \operatorname{Cos}\left(\omega_{2} t-p_{2} \theta+\gamma_{2}\right) \\
\tau=t+t_{0} \\
\eta=\theta+\theta_{0}
\end{gathered}
$$

By substituting (15) and (16) in (14) we will have:

$$
\begin{aligned}
& B_{I I}(\eta, \tau)=B_{1} \operatorname{Cos}\left(\omega_{1} \tau-\omega_{1} t_{0}-p_{1} \eta+p_{1} \theta_{0}\right) \\
& +B_{2} \operatorname{Cos}\left(\omega_{2} \tau-\omega_{2} t_{0}-p_{2} \eta+p_{2} \theta_{0}+\gamma_{2}\right)
\end{aligned}
$$

Now the appropriate values of $t_{0}$ and $\theta_{0}$ should be chosen, to make the two phases become equal.

$$
\left\{\begin{array}{l}
p_{1} \theta_{0}-\omega_{1} t_{0}=0 \\
p_{2} \theta_{0}-\omega_{2} t_{0}+\gamma_{2}=\gamma_{1}
\end{array}\right.
$$

By solving the system we have:

$$
\begin{gathered}
t_{0}=p_{1} \frac{\gamma_{1}-\gamma_{2}}{p_{2} \omega_{1}-p_{1} \omega_{2}} \\
\theta_{0}=\frac{\omega_{1}}{p_{1}} t_{0}=\omega_{1} \frac{\gamma_{1}-\gamma_{2}}{p_{2} \omega_{1}-p_{1} \omega_{2}}
\end{gathered}
$$

If the denominator of the fraction be zero, which is equivalent to $\omega_{s}=0$ (see (21)), the system will not have any solution.

$$
\omega_{s}=\frac{p_{1} \omega_{2}-p_{2} \omega_{1}}{p_{1}+p_{2}}
$$

So we can conclude that $B_{\mathrm{I}}(\theta, t)$ and $B_{\mathrm{II}}(\eta, \tau)$ have the same relation and this is the evidence of the first assert. For the rotor of BDFM, $\omega_{1}$ and $\omega_{2}$ are substituted by $\omega_{s}$, and frequencies are equal, hence denominator never equals zero, other than $\omega_{s}=0$.

According to the stated lemma, losses can be calculated with each arbitrarily selection of $\gamma$ specially $\gamma=0$.

$$
\begin{gathered}
B(\theta, t)=B_{1} \operatorname{Cos}\left(\omega_{1} t-p_{1} \theta\right)+B_{2} \operatorname{Cos}\left(\omega_{2} t-p_{2} \theta\right) \\
\psi_{s}=\left(p_{2}-p_{1}\right) \theta \\
B(\theta)=B_{1} \operatorname{Cos}\left(\omega_{1} t-p_{1} \theta\right)+B_{2} \operatorname{Cos}\left(\omega_{2} t-p_{1} \theta+\psi_{s}\right)
\end{gathered}
$$

Before starting simulation, Eqs. (25-27) are defined for simplicity:

$$
\begin{gathered}
k_{\omega}=\frac{\omega_{2}}{\omega_{1}} \\
k_{B}=\frac{B_{2}}{B_{1}} \\
k_{A}=k_{\omega} k_{B}=\frac{\omega_{2} B_{2}}{\omega_{1} B_{1}}
\end{gathered}
$$




\section{A. Simulation of B-H Hysteresis Loop of the Stator}

For simulation the value of the $B$ and $\frac{d B}{d t}$ are needed:

$$
\begin{gathered}
B(\theta, t)=B_{1} \operatorname{Cos}\left(\omega_{1} t-p_{1} \theta\right)+B_{2} \operatorname{Cos}\left(\omega_{2} t-p_{1} \theta+\psi_{s}\right) \\
\frac{\partial B(\theta, t)}{\partial t}=-B_{1} \omega_{1} \operatorname{Sin}\left(\omega_{1} t-p_{1} \theta\right)-B_{2} \omega_{2} \operatorname{Sin}\left(\omega_{2} t-p_{1} \theta+\psi_{s}\right) \\
=-B_{1} \omega_{1}\left[\operatorname{Sin}\left(\omega_{1} t-p_{1} \theta\right)+k_{A} \operatorname{Sin}\left(k_{\omega} \omega_{1} t-p_{1} \theta+\psi_{s}\right)\right] \\
H(\theta, t)=g^{-1}\left(\frac{\partial B(\theta, t)}{\partial t}\right)+f(B(\theta, t))
\end{gathered}
$$

$f$ and $g$ functions are influenced by the core material and can be determined by experiment. In [17] and [18], to model the hysteresis phenomenon, the presented dynamical system is used, and some polynomial expressions are proposed to estimate the $f$ and $g$ functions. Among them, the most accurate model to estimate the losses in sinusoidal excitation, has a third degree and a fifth degree binomial for "dissipation function" and "restoring function", respectively. In this model, the third and fifth harmonic coefficients of the inductor current, can be calculated directly from the coefficients of the polynomial, by applying sinusoidal voltage to the nonlinear inductor. In spite of useful results for evaluation of the harmonic currents, the accuracy of the model is not enough for precise simulation. Hyperbolic model is one of the most accurate and applicable model for calculation of the restoring function [19]. In general the model can be written as:

$$
H(B)=a B+b \times \sinh (c B)
$$

where $\mathrm{a}, \mathrm{b}$ and $\mathrm{c}$ are constants, which can be determined by the $B-H$ curve for a specific material.

For M5T30 core restoring function can be written as:

$$
H(B)=17.13 B+3.631 \times 10^{-6} \sinh (10.89 B)
$$

For current simulation, this hyperbolic function is selected. For dissipation function, an arbitrary function is suggested, with typical form of dissipation functions of magnetic materials. The form of this function conformed to the dissipation function of the Arnold 4178-S-2 supermalloy core [11] (see Fig. 3).

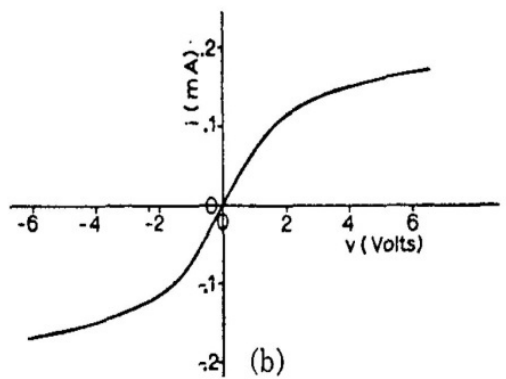

Fig. 3 losses function of the Arnold 4178-S-2 supermalloy

This curve can be divided into three lines, two lines with the same slope in left and right sides, with different heights, and another line, which connects the two, through the origin. The slope of the middle line is four or five times of the two lines. So a hyperbolic tangent and a linear function are proposed for estimation of the function. The numerical values of the function parameters will change final results, but general behavior of hysteresis loops can be simulated by typical parameters. In this case the operating point and variable parameters ranges has been set before running the simulation (Table I).

Table I. The numerical value of the function parameters

\begin{tabular}{|l|l|l|l|l|l|l|}
\hline variable & $B_{1}(\mathrm{~T})$ & $\omega_{1}(\mathrm{~Hz})$ & $k_{\omega}$ & $k_{B}$ & $\psi_{s}$ & time \\
\hline value & 0.72 & $2 \pi \times 50$ & $(0,2]$ & $(0,2]$ & $(0, \pi]$ & $10 \mathrm{~s}$ \\
\hline
\end{tabular}

$$
\begin{aligned}
& B_{1}=0.72 T, \quad \omega_{1}=2 \pi \times 50 H z, \quad k_{\omega} \in(0,2], \quad k_{B} \in(0,2] \\
& t=0: 0.001: 10 \mathrm{sec}, \quad \psi_{s} \in[0, \pi] \\
& f(B)=17.13 B+3.631 \times 10^{-6} \sinh (10.89 B) \\
& g^{-1}(x)=0.01 x+\tanh (0.04 x)
\end{aligned}
$$

Fig. 4 and 5 depict the restoring and dissipation functions.

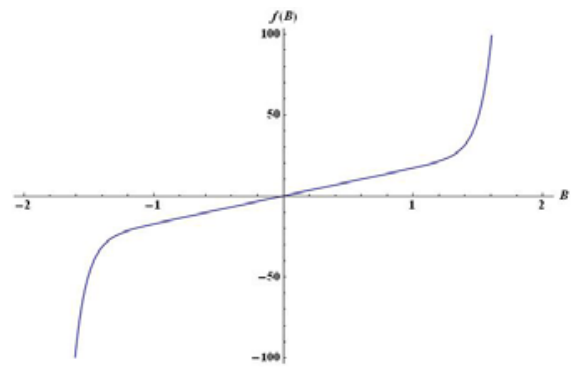

Fig. 4 restoring function

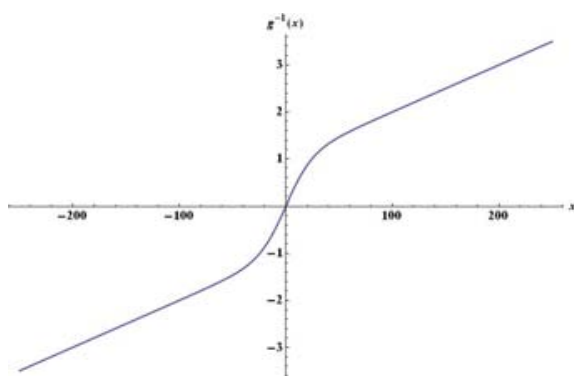

Fig. 5 Dissipation function

The simulated hysteresis loop with $\left(k_{B}, k_{\omega}, \Psi_{s}=\right.$ $(1,0.1,0.157)$ is shown in Fig. 6.

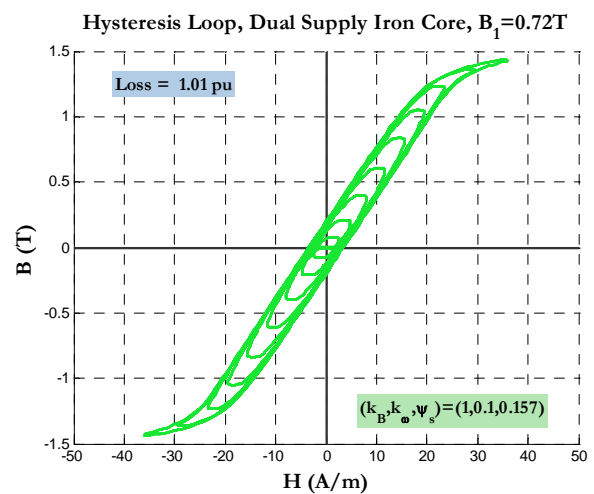

Fig. 6 simulated hysteresis loop

B. Relationship of the Losses with respect to $B_{2}\left(k_{B}\right)$ and $\omega_{2}\left(k_{\omega}\right)$ 
Hysteresis loss can be determined by calculation of variations of the stored energy in the magnetic field [7], through integration of the dot product of magnetic flux density $(B)$ and field intensity $(H)$ on a specific time period and volume. Assuming that the directions of the vectors are the same we can write:

$$
W=\int_{V}\left[\int_{T} H \cdot d B\right] d v=\int_{V}\left[\int_{T} H \frac{\partial B}{\partial t} d t\right] d v
$$

Using (35), hysteresis losses in the rotor and stator of the BDFM can be calculated.

\section{1) Rotor Hysteresis Loss}

Hysteresis loss for a sinusoidal field is well known. One of the practical equations is Estein Metz equation [6]:

$$
W_{h}=K_{H} B_{m}^{n} f \quad\left(W / m^{3}\right)
$$

where $B_{m}$ is the maximum flux density and $\mathrm{n}$ and $K_{H}$ are constant coefficients. For simplicity, the $n=2$ estimate is used, which corresponds to the linear region of the magnetization curve. So, (36) can be written as:

$$
P_{r, h}=\frac{K_{h}}{2 \pi} B_{m}^{2} \omega_{s}=\kappa_{r} B_{m}^{2} \omega_{s}
$$

where $\kappa_{r}$ is the constant of the rotor hysteresis losses. Because the rotor has one frequency, the hysteresis losses can be calculated easily:

$$
\begin{gathered}
B_{r}(\varphi)=B_{1} \operatorname{Cos}\left(\omega_{s} t+p_{1} \varphi\right)+B_{2} \operatorname{Cos}\left(\omega_{s} t+p_{2} \varphi\right) \\
=B_{1} \operatorname{Cos}\left(\omega_{s} t+p_{1} \varphi\right)+B_{2} \operatorname{Cos}\left(\omega_{s} t+p_{1} \varphi+\psi_{r}\right) \\
\psi_{r}=\left(p_{2}-p_{1}\right) \varphi \\
B_{r}(\varphi)=\left[B_{1}^{2}+B_{2}^{2}+2 B_{2} B_{1} \operatorname{Cos}\left(\psi_{r}\right)\right]^{1 / 2} \\
\times \operatorname{Sin}\left(\omega_{s} t+p_{1} \varphi+\operatorname{tg}^{-1}\left(\frac{k_{B} \operatorname{Sin} \psi_{r}}{1+k_{B} \operatorname{Cos} \psi_{r}}\right)\right)
\end{gathered}
$$

The pick of hysteresis loop depends only on the amplitude of the $B_{r}(\varphi)$.

$$
P_{r, h}=\kappa_{r}\left[B_{1}^{2}+B_{2}^{2}+2 B_{2} B_{1} \operatorname{Cos}\left(\psi_{r}\right)\right]\left|\omega_{s}\right|
$$

by averaging the above equation on $\varphi$, we have:

$$
\bar{P}_{r, h}=\kappa_{r}\left[B_{1}^{2}+B_{2}^{2}\right]\left|\omega_{s}\right|=\kappa_{r} B_{1}^{2}\left[1+k_{B}^{2}\right]\left|\omega_{s}\right|
$$

On the other hand:

$$
\omega_{s}=\frac{p_{1} \omega_{2}-p_{2} \omega_{1}}{p_{1}+p_{2}}=\frac{k_{\omega}-k_{p}}{1+k_{p}}
$$

So,

$$
\bar{P}_{r, h}=\kappa_{r} B_{1}^{2}\left[1+k_{B}^{2}\right] \frac{\left|k_{\omega}-k_{p}\right|}{1+k_{p}}\left(W / m^{3}\right)
$$

\section{2) Stator Hysteresis Loss}

To calculate this loss, numerical simulation is proposed. The instantaneous power dissipation is calculated in each $\theta$ angel of stator core first, and the result is averaged over time, then the angel $\theta$.

The hysteresis loss can be written as:

$$
\bar{P}_{s, h}(\theta)=\frac{1}{T} \int_{T} H \frac{\partial B}{\partial t} d t=\operatorname{mean}(H \dot{B})\left(W / m^{3}\right)
$$

where $\bar{P}_{s, h}(\theta)$ is stator hysteresis loss density, as function of the stator angle.

Fig. 7 depicts the result of numerical simulation of the stator hysteresis loss variations with respect to frequency ratio $\left(k_{\omega}\right)$ and flux ratio $\left(k_{B}\right)$.

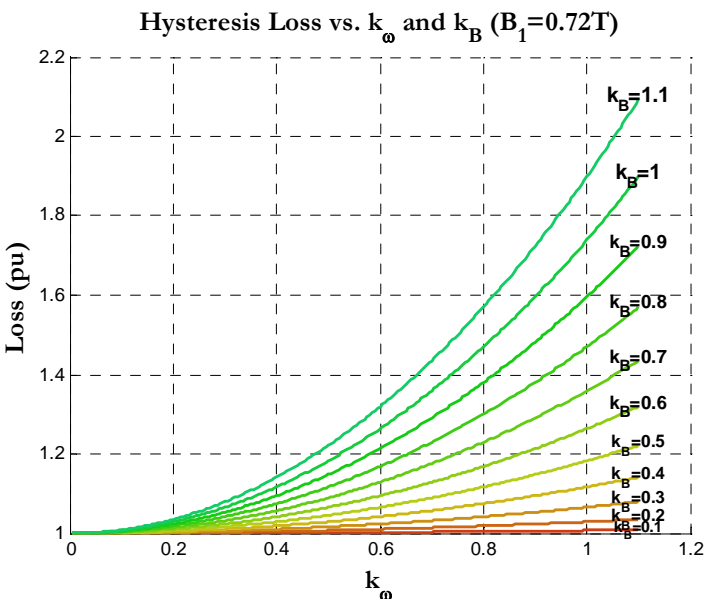

Fig. 7 BDFM hysteresis losses toward different frequency ratio and stators rotary fields ratio $(\mathrm{B}=0.72 \mathrm{~T})$

For this simulation $B_{1}=0.72 T$ is set, where the peak value of the machine field at $k_{\omega}=1$ and $k_{B}=1$ is set on the knee point of the $B-H$ curve. At this operating point, loss value can be interpolated as quadratic function of frequency ratio. However, if the field increases, the core will be saturated. In spite of that, it can be seen from the Fig. 8, which is for $B=1 T$, that the loss contours are parabolic again.

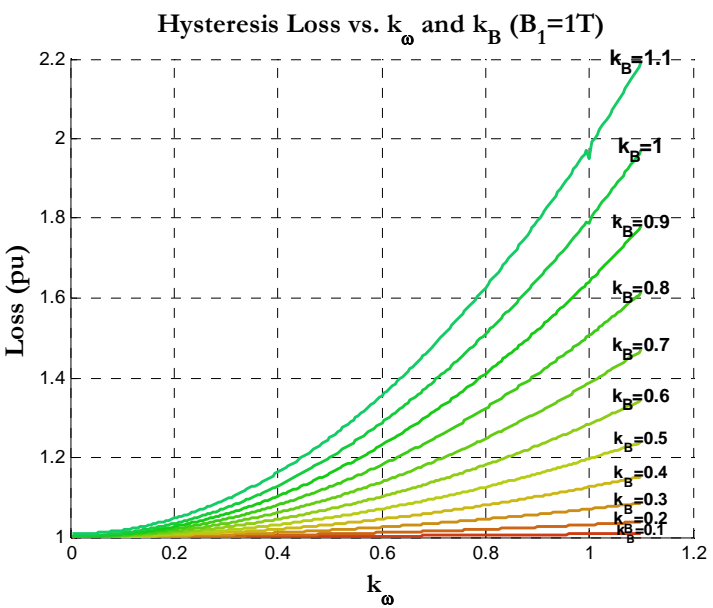

Fig. 8 BDFM hysteresis losses toward different frequency ratio and stators rotary fields ratio $(B=1 \mathrm{~T})$

We can see:

$$
\bar{P}_{s, h}=\kappa_{s} B_{1}^{2} \omega_{1}\left(1+\zeta_{h} k_{\omega}^{2}\right)
$$

In this parabolic formulation, $\zeta_{h}$ varies by $k_{B}$. To find the relationship between $\zeta_{h}$ and $k_{B}$, the flux density is regulated at $0.72(B=0.72)$, and for some different values of $k_{B}$ the simulation is repeated. Table II shows the variable changes: 
Table II. hysteresis losses toward field variations

\begin{tabular}{|c|c|c|c|c|c|c|c|c|c|c|c|}
\hline$k_{B}$ & 0.0 & 0.1 & 0.2 & 0.3 & 0.4 & 0.5 & 0.6 & 0.7 & 0.8 & 0.9 & 1.0 \\
\hline$\zeta_{h}$ & 0.0 & 0.0074 & 0.0294 & 0.066 & 0.117 & 0.183 & 0.264 & 0.360 & 0.471 & 0.597 & 0.739 \\
\hline
\end{tabular}

Fig. 9 portrays the graph of data of the Table II in logarithmic scale. Again the relationship is quadratic, similar to the previous quadratic relationship of loss and frequency ratio.

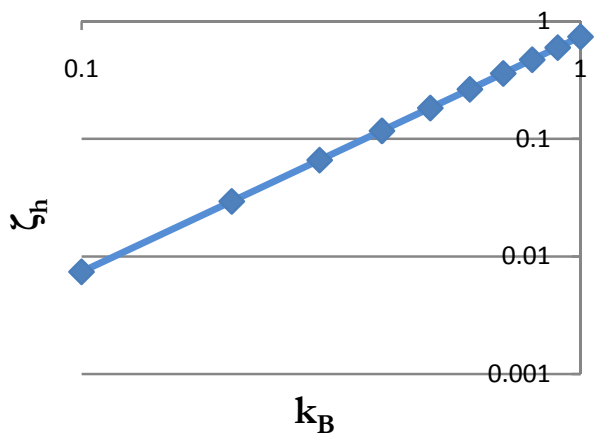

Fig. 9 Data of the Table II.

Interpolation of data results in:

$$
\begin{gathered}
\zeta_{h}=\alpha_{h} k_{B}^{2}, \quad \alpha_{h}=0.74 \\
\bar{P}_{s, h}=\kappa_{s} B_{1}^{2} \omega_{1}\left(1+\alpha_{h} k_{A}^{2}\right) \quad\left(W / m^{3}\right)
\end{gathered}
$$

It can be seen that the relationship is quadratic again. The coefficient of quadratic function is assumed linearly dependant on $\omega_{1}$ and proportional to square of $B_{l}$. Assigning power of two for $B_{l}$ is valid, since the peak value of $B_{1}$ is far enough from the knee point of $B-H$ curve, when $B_{2}$ is equal to zero; and the $B-H$ curve is assumed linear.

\section{Conclusions}

In this paper, an analytical hysteresis modelling approach was presented for brushless doubly fed induction machines. The base model was a dynamical system, with dissipation and restoring function characteristics. This model was used for calculation of hysteresis loss in the stator of BDFM, where two different frequency fields exist. After simulation of hysteresis loops of the field, the hysteresis loss was calculated and subsequent curves were fitted on quadratic functions, resulting in quadratic relationship between stator's hysteresis loss, and multiplication of control winding supply frequency $\left(\omega_{2}\right)$ and field amplitude $\left(B_{2}\right)$.

\section{References}

[1] N. Mohan, T. M. Undeland, and W. P. Robbins, Power Electronics: Converters, Applications and Design, 2nd ed. New York: Wiley, 1991.

[2] R.A. McMahon, P.C. Roberts, X. Wang, P.J. Tavner. "Performance of BDFM as generator and motor", IEE Proc. Electr. Power Appl., Vol.153, No.2, March 2006.

[3] A. C. Ferreira and S. Williamson, "Time-Stepping FiniteElement Analysis of Brushless Doubly Fed Machine Taking Iron Loss and Saturation into Account," IEEE
Trans. on Industry Applications, vol. 35, no. 3, pp. 583588, May/June 1999.

[4] O. I. Butler and M. R. Sarna, "Relaxation methods applied the problem of ac magnetizaiion of ferromagnetic laminate," in Proc. IEE, London, 1951, pp. 389-398.

[5] G. L. Shurtz, "Time dependence of the magnetic field in a rectangular toroid," in Conf. Rec. 10th Midwest Symp. on Circuit Theory, Lafayette, Ind., 1966.

[6] C. P. Steinmetz, "On the law of hysteresis," AIEE Transactions, vol. 9, pp. 3-64, 1892,Reprinted under the title "A Steinmetz contribution to the ac power revolution ", introduction by J.E. Brittain, in Proceedings of the IEEE 72(2), 1984, pp. 196-221.

[7] S. Moosavi, "Calculation of Harmonic Losses in Transformer's Core", University of Tehran, Master Thesis, 2008.

[8] Motorsoft INC. User Manual for PC-SRD 4.7. Switched reluctance motor simulation software.

[9] Y. Chen and P. Pillay, "An Improved Formula for Lamination Core Loss Calculations in Machines Operating with High Frequency and High Flux Density Excitation," Conf. Rec. IEEE-IAS Annu. Meeting, vol. 2, pp. 759-766, October 2002.

[10] L. A. Zadeh and C. A. Desoer, Linear System Theory, The State Space Approach. New York: McGraw-Hill, 1963.

[11] L. O. Chua and K. A. Stromsmoe, "Lumped-Circuit Models for Nonlinear Inductors Exhibiting Hysteresis Loops," IEEE Trans. on Circuit Theory, vol. CT-17, no. 4, pp. 564-574, November 1970.

[12] V. Volterra, Theory of Functionals and of Integral and Integro-Differential Equations. New York: Dover, 1959.

[13] H. D. Block, "Periodic solutions of forced systems having hysteresis," IRE Trans. Circuit Theory, vol. CT-37, pp. 423-431, December 1960.

[14] R. J. P. DeFigueiredo, "Forced oscillations in nonlinear systems with hysteresis," IRE Trans. Circuit Theory, vol. CT-12, pp. 52-58, March 1965.

[15] J. A. Resh, "Towards general simulation of ferromagnetic devices," in Pioc. 18th Midwest Symp. on Circuit Theory, Austin, Texas, 1969, pp. V.2.1-V.2.7.

[16] Paul C. Roberts, "A study of brushless doubly-fed (induction) machines," University of Cambridge, Ph.D. dissertation 2005.

[17] S. Prusty and M.V.S. Rao, "New method for predetermination of true saturation characteristics of transformers and nonlinear reactors," Proc. IEE, vol. 127, pp. 106-110, 1980.

[18] S. Prusty and M. V. S. Rao, "A Novel Approach for Predetermination of Magnetization Characteristics of Transformers Including Hysteresis," IEEE Transactions on Magnetics, vol. MAG-20, no. 4, pp. 607-612, July 1984.

[19] M. Khelil and M. Elleuch, "Modeling of Air-gaps of Overlapped Joints in Three Phase Transformer Iron Core for using by FEM," in 6th International Multi-Conference on Systems, Signals and Devices, 2009. 